\title{
Association of colorectal cancer and GIST tumors - A diagnostic and therapeutic challenge: A case report
}

\author{
Ciprian Cirimbei ${ }^{1,2 \star}$, Vlad Rotaru ${ }^{1}$, Madalina Radu ${ }^{3}$ and Simona Elena Cirimbei ${ }^{1,2}$ \\ ${ }^{1}$ Department of Surgical Oncology, Institute of Oncology "Prof. Dr. Al. Trestioreanu" Bucharest, Romania. \\ ${ }^{2}$ "Carol Davila" University of Medicine and Pharmacy, Bucharest, Romania. \\ ${ }^{3}$ Department of Pathology, Institute of Oncology "Prof. Dr. Al. Trestioreanu" Bucharest, Romania.
}

Accepted 6 May, 2020

\begin{abstract}
The association between colorectal cancer and gastrointestinal stromal (GIST) tumors is increasingly cited in medical literature as an argument in the plea for full exploration of patients with digestive malignancies. The coexistence of the two lesions is frequently diagnosed randomly, pre- or intraoperatively, and may raise diagnostic and therapeutic problems. We present a case of association of a recto-sigmoid junction cancer with a GIST gastric tumor with full imaging and anatomo-pathological description, as well as its surgical and oncological management. Pre-, intra- and postoperative histopathological examinations, correlated with immunohistochemical tests, are essential to determine the key points of the therapeutic strategic.
\end{abstract}

Keywords: Colorectal cancer, GIST tumors, surgical and oncologic management.

*Corresponding author. E-mail: ccirimbei@gmail.com. Tel: +40 722268749, +40 212271000. Fax: +40 213183262.

\section{INTRODUCTION}

The synchronous occurrence of tumoral lesions in the digestive tract is an association characterised by increasing incidence, as evidenced by data reported in the literature. There are multiple possible explanations, which have been linked particularly to carcinogenetic mechanisms and the intrinsic and extrinsic triggers of neoplastic processes (Sammarco et al., 2004; Paschalis and Nikolaidou, 2015). The incidence of gastrointestinal stromal tumours, neoplastic mesenchymal lesions with malignant potential, has been estimated around 1 to $1.5 / 100.000$ per year, accounting for less than $1 \%$ of gastrointestinal tumours. They develop in the interstitial cells of Cajal, located in the muscular coat, inside and around the myenteric plexus, showing both myogenic and neural differentiation, and are characterised by an over expression of tyrosine kinase (KIT) receptors (Mazur and Clark, 1983; Siehl and Thiel, 2007).

The coexistence of GIST tumours and colorectal cancer, the third cause of cancer-related death reported in the USA, is often inadvertently diagnosed pre- or intraoperative (Melis et al., 2007; Nemes et al., 2012), and can, potentially raise problems in terms of surgical and oncologic management. Various authors have reported for this association a frequency rate ranging from 2.95 to $33 \%$ (Arnogiannaki et al., 2010; Agaimy et al., 2006).

We present here a case in which the association was diagnosed during the imaging investigations for a colorectal cancer, which required the modification of the diagnosis algorithm to ensure treatment optimisation.

\section{Case report}

Sixty-year old female patient, with a medical history of total laparoscopic hysterectomy performed 7 years before for uterine fibromatosis, was admitted to hospital for abdominal and pelvic pain accompanied by rectorhagia, the onset of which had occurred approximately 3 months previously.

The colonoscopy revealed the presence of a haemorrhagic ulcero-vegetant and partially stenotic tumour, located approximately 18 to $20 \mathrm{~cm}$ from EAS (Figure 1a and $b$ ).

An additional full CT investigation of the chest, abdomen and pelvis was performed, which confirmed the 


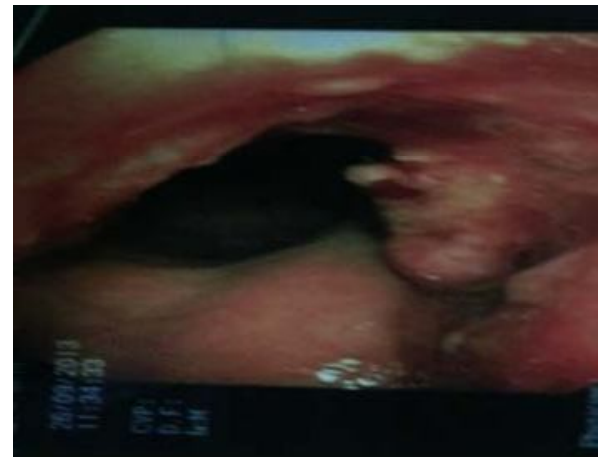

$\mathbf{a}$

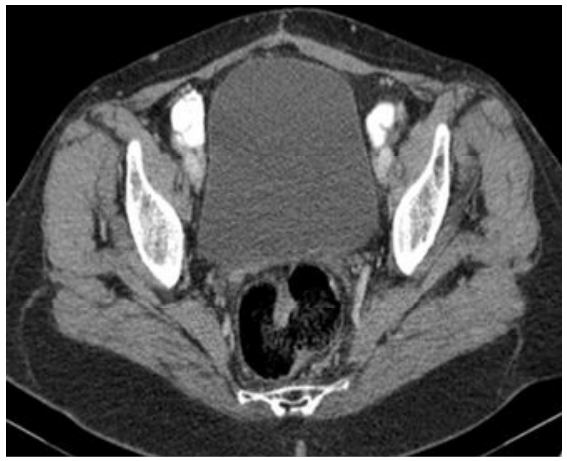

b

Figure 1. Rectosigmoid tumour with endoscopic (a) and CT (b) appearance.

rectosigmoid tumour with satellite adenopathies, without visceral metastases or evidence of carcinomatosis, and showed a concomitant gastric fundus lesion measuring approximatively 3 to $4 \mathrm{~cm}$, with intramural development and peripheral calcification, protruding in the gastric lumen. The gastric tumour has hypodense and nonhomogenous structure, causing the retraction of the adjacent gastric wall, revealing peripheral solid density and calcifications and a central area of necrosis. After the administration of contrast agent, the peripheral areas show intense uptake of the contrast substance.

The endoscopic investigation performed confirms the intra-gastric lesion, describing a well-defined subcardial tumour structure, measuring approximately 2 to $3 \mathrm{~cm}$, covered by mucosa with normal appearance (Figure 2a and $b$ ).

Surgery was performed, confirming the presence of the 2 lesions, gastric and rectosigmoid, followed by modelling gastric resection at the level of the greater gastric curvature (after performing a prior extemporaneous histopathologic examination that raised the suspicion of gastric GIST). In correlation, a rectosigmoid resection with total mesorectal excision is performed, followed by termino-terminal colorectal anastomosis. The postoperative evolution was favourable, without complications.

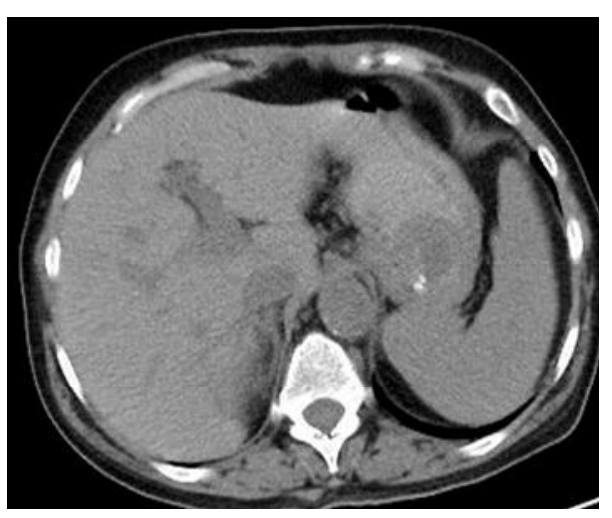

$\mathbf{a}$

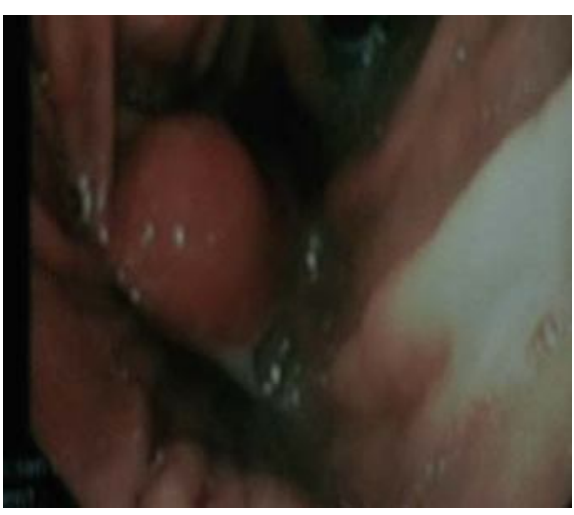

b

Figure 2. Gastric tumour with CT (a) and endoscopic (b) appearance.

\section{Pathological findings}

The findings of paraffin histopathology described the presence of a gastric tumour measuring $2.5 / 3.5 \mathrm{~cm}$, with greyish-white colouring, encapsulated, of medium consistency, with areas of calcification, presenting normal margins of resection measuring $1.5 \mathrm{~cm}$, raising the suspicion of gastric GIST; IHC tests are recommended. The colon tumour is a moderately differentiated adenocarcinoma (G2), with superficial areas of necrosis, infiltrating the muscle layer of the intestinal wall, with normal resection limits at more than $7 \mathrm{~cm}$ from the tumour; the 7 lymph nodes identified were characterised by sinus histiocytosis pT3NOMO (Figure 3a and b). 


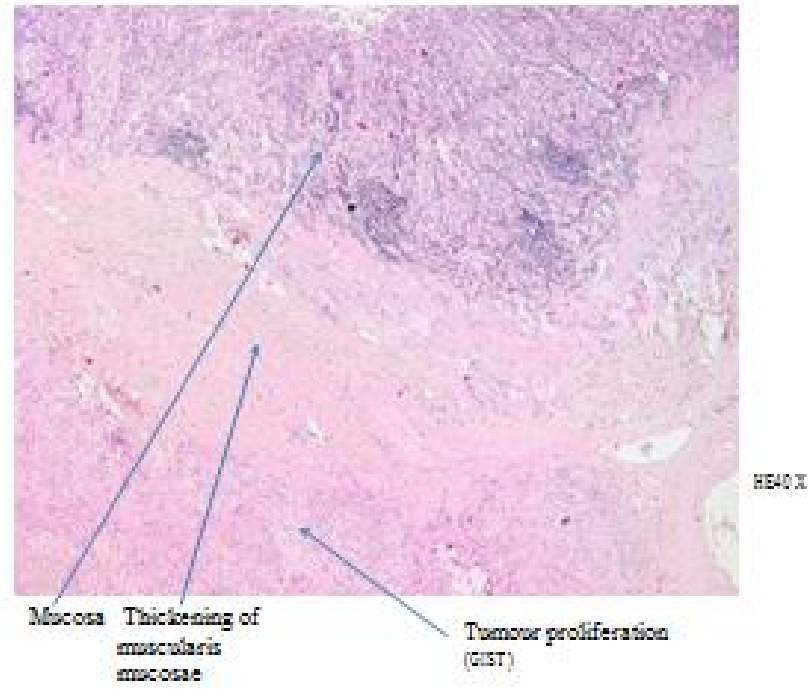

$\mathbf{a}$

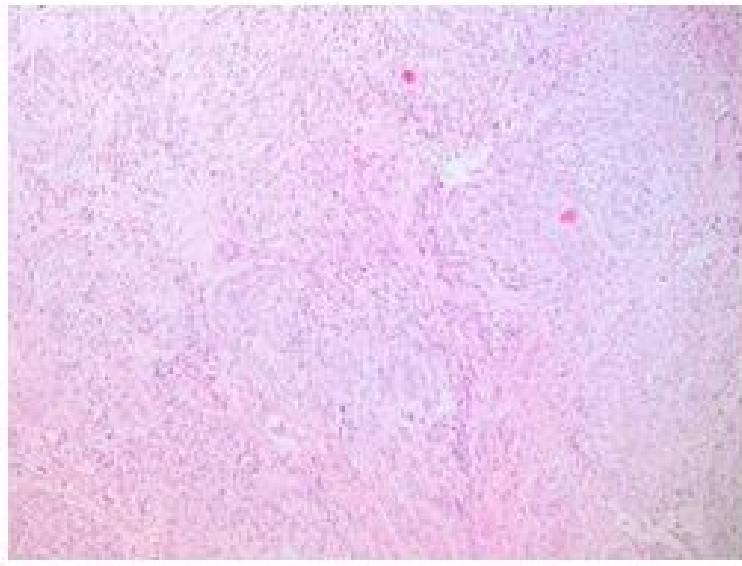

Tvenox proliferation with distribstion in favciewles of warious lengthe, insersecting at differest angles, comprising of fusiform cells with elongased

b

Figure 3. Gastric tumour with histologic appearance at HE 40X (a) and HE 100X (b). a Mucosa-Thickening of muscularis mucosae Tumour proliferation (GIST). b Tumour proliferation with distribution in fascicules of various lengths, intersecting at different angles, comprising of fusiform cells with elongated nuclei.

The histologic and immunohistochemical re-evaluation of the gastric lesion indicates tumour proliferation at the level of the gastric submucosa, consisting in fusiform cells with pale eosinophilic cytoplasm, arranged in intersecting fascicules, with low mitotic activity (2 mitoses/50 HPF) and unidentified intravascular invasion (LVI). The immunohistochemical tests performed indicate that the tumour proliferation has positive diffusion for CD117, DOG-1, CD34 and negative for Smooth Muscle Actin. The final histopathologic diagnosis is gastrointestinal stromal tumour (GIST), with low histologic grade, pT2NOMO, prognosis group 2 (WHO 2010), unidentified LVI, KIT positive (Figure $4 \mathrm{a}$ and b, Figure 5a and $b$ ).

Additionally, in order to exclude a GIST component in the colon tumour, the related immunohistochemical tests were performed, showing: CK20-positive diffuse in the tumour cells, P53-positive $-75 \%$ in the tumour cells, EGFR - zonal positive in tumour cells, KI67-positive $55 \%$ in the tumour cells, findings that support the diagnosis of adenocarcinoma.

\section{DISCUSSION}

The synchronous occurrence of lesions that was identified resulted in diagnosis and treatment problems. The intraoperative resolution of the gastric lesion as a suspected GIST lesion (following the extemporaneous histopathologic examination), with a potential benign behaviour, required the limitation of the surgical sanction to a $\mathrm{RO}$ resection (modelling gastric resection type), while the colon lesion, suggestive of colorectal cancer, necessitated a rectocolectomy with adjacent lymphadenectomy. It becomes essential to determine the dominant neoplastic lesion in light of the therapeutic approach to be followed. The complete treatment of both lesions after postoperative staging requires in addition to the surgical sanction, a specific multimodal oncologic therapy approach to be determined based on the postoperative staging and specific histologic, immunohistochemical and genetic markers. Thus, the oncologist was faced with the dilemma of selecting the appropriate chemotherapy regimen.

An essential step to determine the prognosis and treatment of a localised GIST tumour is to identify the 3 main prognosis factors: tumour size (positive prognosis < $5 \mathrm{~cm}$ ), tumour location (gastric localisation being more favourable as compared with recto-colic location) and number of mitoses (poor prognosis in case of increased mitotic activity $>5$ mitoses per 50 high-power microscopic fields) (Akahoshi and Oya, 2010; Roggin and Posner, 2012; Reddy and Fleshman, 2006). Once identified, these parameters allow for the classification of lesions in a certain risk category (very low, low, moderateintermediary or high) in terms of potential for relapse. A significant prognosis value is also represented by the intraperitoneal tumour rupture and positive margins of resection, which are added to the high-risk category. Consequently, patients with a lesion classified in the high or moderate-intermediary risk category can benefit from adjuvant first-line treatment with imatinib, in various doses, or second or third line medication -sunitinib, regorafenib, sorafenib, dasatinib or nilotinib (ESMO, 2012).

Morphologic and histopathologic characteristics 


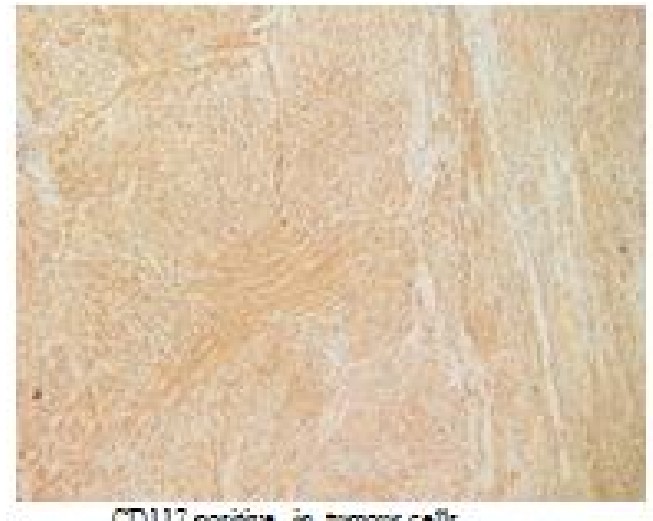

CD117 positive in tzenors cells

$\mathbf{a}$

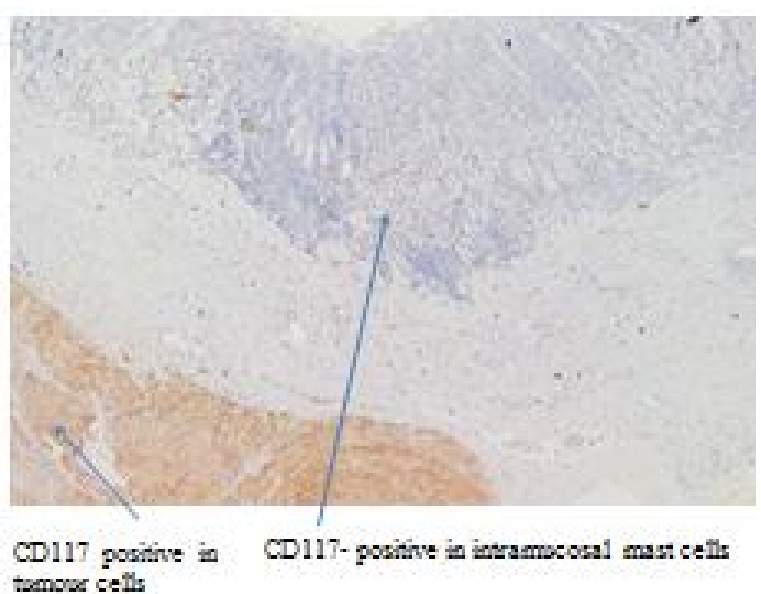

b

Figure 4. Immunohistochemistry of gastric tumour: CD117 (c-kit) positive in tumour cells (a) and CD117- positive intramucosal mast cells (b).

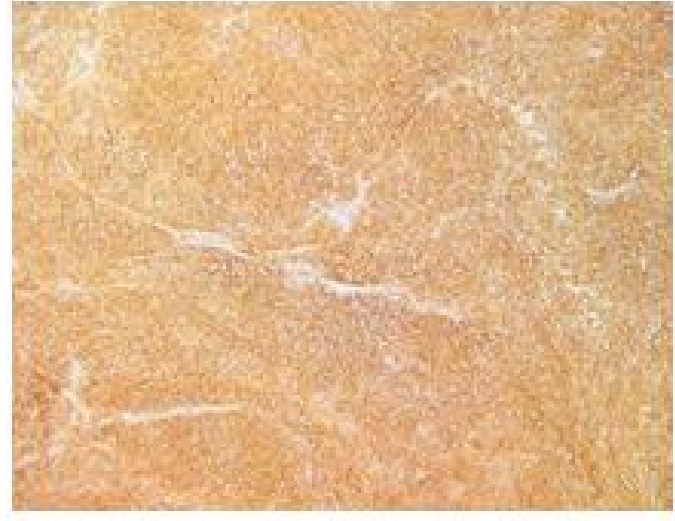

CD34-positive in temoxe cells

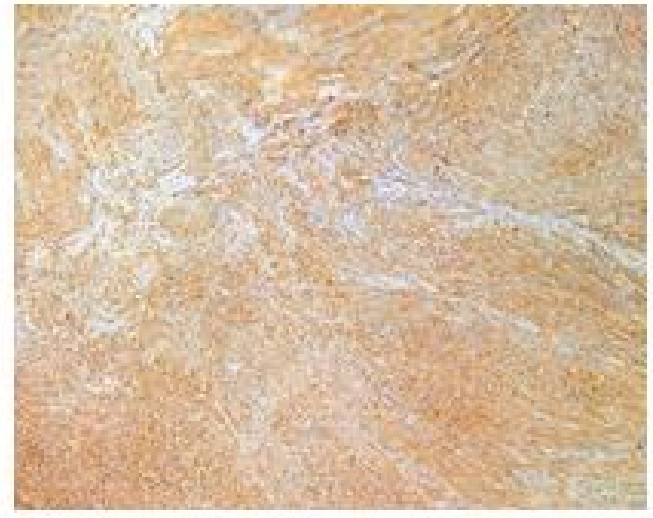

DOG 1-positive in tsemorx cells

Figure 5. Immunohistochemistry of gastric tumour: CD34-positive (a) and DOG1-positive (b) tumour cells.

included a class 2 GIST (size between 2 and $5 \mathrm{~cm}$, gastric location, less than 5 mitoses/50 HPF, without intraperitoneal tumour rupture and negative margins of resection), with a very low risk of relapse and metastasis. As a result, first-line chemotherapy with imatinib is not indicated, due to the fact that this type of lesion staging requires only imaging monitoring (endoscopy and CT).

With regard to the colic lesion, based on the morphologic and histopathologic characteristics it was classified as IIA TNM Stage (pT3NOM0). Following the completion of the surgical sequence, adjuvant chemotherapy was necessary to prevent relapse, the only arguments consisting in the incomplete degree of obstruction and moderate G2 differentiation. While being subject to debate, the chemotherapy option was determined after considering studies that reported an increase of survival rates for 2 to $4 \%$ patients. The patient underwent the traditional regimen with administration of 5 -FU in 6 rounds, as the only chemotherapy for the princeps colic lesions, while the GIST lesion did not require any specific adjuvant therapy.

Immediate and long-term monitoring for 3 years, which included regular endoscopic examinations and CT investigations of the chest and abdomen regions, alongside tumour markers, has shown no relapse or distant metastases.

\section{Conclusions}

The relatively increasing incidence of the association between colorectal cancer and GIST tumours requires a complete evaluation of patients with digestive neoplasia, including a case study performed by a multidisciplinary 
team comprising of surgeons, anatomical- pathologists, oncologists, radiologists and gastroenterologists, providing a full description of lesions for the purpose of determining the therapeutic algorithm. Pre-, intra- and postoperative histopathological examinations, correlated with immunohistochemical tests, are essential to determine the key points of the therapeutic strategic.

\section{REFERENCES}

Agaimy A, Wunsch PH, Sobin LH, Lasota J, Miettinen M, 2006. Occurrence of other malignancies in patients with gastrointestinal tumors. Semin Diagn Pathol, 23(2): 120-129.

Akahoshi K, Oya M, 2010. Gastrointestinal stromal tumor of the stomach: How to manage? World J Gastrointest Endosc, 2(8): 271277.

Arnogiannaki N, Martzoukou I, Kountourakis P, Dimitriadis E, Papathanasaki A, Nastoulis E, Gazalidou M, Fida A, Apostolikas N, Agnantis NJ, 2010. Synchronous presentation of GISTs and other primary neoplasms: a single centre experience. In vivo, 24(1): 109115.

ESMO / European Sarcoma Network Working Group, 2012. Gastrointestinal stromal tumors: ESMO Clinical Practice Guidelines for diagnosis, treatment and follow-up. Ann Oncol, 23(Suppl 7): vii49vii55.

Mazur MT, Clark HB, 1983: Gastric stromal tumors. Reappraisal of histogenesis. Am J Surg Pathol 7(6): 507-519.

Melis M, Choi EA, Anders R, Christiansen P, Fichera A, 2007. Synchronous colorectal adenocarcinoma and gastrointestinal stromal tumor (GIST). Int J Colorectal Dis 22(2): 109-114.

Nemes C, Rogojan L, Surdea-Blaga T, Seicean A, Dumitrascu DL, Ciuce C, 2012. Gastrointestinal stromal tumor (GIST) associated with synchronous colon adenocarcinoma - A case report. J Gastrointestin Liver Dis, 21(1): 101-103.

Paschalis G, Nikolaidou A, 2015. Colon adenocarcinoma associated with synchronous extramural gastrointestinal stromal tumor (GIST) of the lleum. Am J Case Rep, 16: 837-839.

Reddy RM, Fleshman JW, 2006. Colorectal Gastrointestinal Stromal Tumors: A Brief Review. Clin Colon Rectal Surg, 19(2): 69-77.

Roggin KK, Posner MC, 2012. Modern treatment of gastric gastrointestinal stromal tumors. World J Gastroenterol, 18(46): 6720-6728.

Sammarco I, Capurso G, Coppola L, Bonifazi AP, Cassetta S, Delle Fave G, Carrara A, Grassi GB, Rossi P, Sette C, Geremia R, 2004. Expression of the proto-oncogene c-KIT in normal and tumour tissues from colorectal carcinoma patients. Int J Colorectal Dis, 19(6): 545553.

Siehl J, Thiel E, 2007. C-kit, GIST and imatinib. Recent Results Cancer Res, 176: 145-151.
Citation: Cirimbei C, Rotaru V, Radu M , Cirimbei SE, 2020. Association of colorectal cancer and GIST tumors - A diagnostic and therapeutic challenge: A case report. Int Res J Med Med Sci, 8(2): 25-29. 